\title{
Filtered Hopf algebras and counting geodesic chords
}

\author{
Urs Frauenfelder • Felix Schlenk
}

\begin{abstract}
We prove lower bounds on the growth of certain filtered Hopf algebras by means of a Poincaré-Birkhoff-Witt type theorem for ordered products of primitive elements. When applied to the loop space homology algebra endowed with a natural length-filtration, these bounds lead to lower bounds for the number of geodesic paths between two points. Specifically, given a closed manifold $M$ whose universal covering space is not homotopy equivalent to a finite complex and whose fundamental group has polynomial growth,
\end{abstract}

for any Riemannian metric on $M$, any pair of non-conjugate points $p, q \in M$, and every component $\mathcal{C}$ of the space of paths from $p$ to $q$, the number of geodesics in $\mathcal{C}$ of length at most $T$ grows at least like $e^{\sqrt{T}}$.

Using Floer homology, we extend this lower bound to Reeb chords on the spherisation of $M$, and give a lower bound for the volume growth of the Reeb flow.

Mathematics Subject Classification (2000) Primary 16T05; Secondary 37B40 . $37 \mathrm{C} 35 \cdot 53 \mathrm{D} 25 \cdot 57 \mathrm{~T} 25$

UF partially supported by the Basic Research fund 2013004879 of the Korean government. FS partially supported by SNF grant 200020-144432/1.

U. Frauenfelder

Department of Mathematics and Research Institute of Mathematics, Seoul National University,

Seoul, Korea

e-mail: frauenf@snu.ac.kr

F. Schlenk $(\bowtie)$

Institut de Mathématiques, Université de Neuchâtel, Neuchâtel, Switzerland

e-mail: schlenk@unine.ch 


\section{Introduction and main results}

A closed connected manifold $M$ is said to be of finite type if its universal cover $\widetilde{M}$ is homotopy equivalent to a finite $\mathrm{CW}$-complex. Equivalently, $M$ is not of finite type if there exists $k \in\{2, \ldots, \operatorname{dim} M\}$ such that $H_{k}(\widetilde{M})$ is not finitely generated. Let $\mathfrak{m}=\mathfrak{m}(M)$ be the minimal such $k$.

A finitely generated group $G$ is called polycyclic if it admits a subnormal series with cyclic factors. Moreover, $G$ is virtually polycyclic if it has a polycyclic subgroup of finite index.

Choose a point $p \in M$ and let $\Omega_{0} M$ be the space of contractible continuous loops in $M$ based at $p$. For $T>0$ denote by $\Omega_{0}^{T} M$ the space of contractible piecewise smooth loops based at $p$ whose length (with respect to a fixed Riemannian metric on $M$ ) is at most $T$. The inclusions $\iota^{T}: \Omega_{0}^{T} M \hookrightarrow \Omega_{0} M$ induce the maps $\iota_{*}^{T}: H_{*}\left(\Omega_{0}^{T} M\right) \rightarrow$ $H_{*}\left(\Omega_{0} M\right)$ in homology. Note that the image $\iota_{*}^{T} H_{*}\left(\Omega_{0}^{T} M\right)$ is the part of the homology of $\Omega_{0} M$ that is generated by cycles made of piecewise smooth loops of length $\leq T$.

We say that a real-valued function $f$ defined on $\mathbb{N}$ or on $\mathbb{R}_{>0}$ grows at least like $e^{\sqrt{T}}$ if there exists a constant $c>0$ such that $f(T) \geq c e^{\sqrt{T}}$ for all large enough $T$.

Theorem 1.1 Let $M$ be a closed connected manifold that is not of finite type and has virtually polycyclic fundamental group. Assume also that there exists a field $\mathbb{F}$ such that $H_{\mathfrak{m}}(\widetilde{M} ; \mathbb{F})$ is infinite-dimensional. Then the function of $T$

$$
\operatorname{dim} \iota_{*}^{T} H_{*}\left(\Omega_{0}^{T} M ; \mathbb{F}\right)
$$

grows at least like $e^{\sqrt{T}}$.

Remark 1.2 (i) The assumption that $H_{\mathfrak{m}}(\tilde{M} ; \mathbb{F})$ is infinite-dimensional is equivalent to the assumption that $\pi_{\mathfrak{m}}(M) \otimes \mathbb{F}$ is infinite-dimensional (see Sect. 5.4 for the proof).

(ii) During the proof we shall see that $H_{\mathfrak{m}-1}\left(\Omega_{0} M ; \mathbb{F}\right)$ is infinite-dimensional and that

$$
\operatorname{dim} H_{(\mathfrak{m}-1) n}\left(\Omega_{0} M ; \mathbb{F}\right) \geq q(n) \text { for } n \geq 2,
$$

where $q(n)$ is the number of partitions of $n$ into distinct parts. The fact that this function grows like $e^{\sqrt{n}}$ explains the lower bound in Theorem 1.1.

(iii) Our assumption that there exists a field $\mathbb{F}$ such that $H_{\mathfrak{m}}(\widetilde{M} ; \mathbb{F})$ is infinitedimensional not only plays an important role in our proof, but may well be essential. Indeed, in Sect. 6 we first generalize Theorem 1.1 to compact simplicial complexes embedded in some Euclidean space, and then show that a simplicial complex $K$ homotopy equivalent to the mapping torus of a degree- $p$ map of $S^{n}$ (with $p$ prime and $n \geq 2$ ) has the following properties: All assumptions of Theorem 1.1 are met besides for $H_{\mathfrak{m}}(\widetilde{K} ; \mathbb{F})$ being zero- or one-dimensional for every field $\mathbb{F}$, but $\iota_{*}^{T} H_{*}\left(\Omega_{0}^{T} K\right)$ is a free Abelian group whose rank grows not more than linearly with $T$.

For manifolds of non-finite type it is still possible that the rank of $\iota_{*}^{T} H_{*}\left(\Omega_{0}^{T} M\right)$ grows exponentially (cf. the Question in [27, p. 289] and the discussion in [8, §7.1]). 
Example 1.3 There are many manifolds meeting the assumptions of Theorem 1.1. For instance, take closed connected manifolds $M_{1}$ and $M_{2}$ of the same dimension, with $\pi_{1}\left(M_{1}\right)$ an infinite polycyclic group and $M_{2}$ simply-connected and not a homotopy sphere. For simplicity we also assume that $H_{2}\left(M_{2}\right) \neq 0$. Consider the connected sum $M=M_{1} \# M_{2}$. Then $\pi_{1}(M)=\pi_{1}\left(M_{1}\right)$ is polycyclic. The universal cover $\widetilde{M}$ is the universal cover $\widetilde{M}_{1}$ with one copy of $M_{2}$ attached for each element of $\pi_{1}\left(M_{1}\right)$. Since $\pi_{1}\left(M_{1}\right)$ is infinite, $M$ is not of finite type, and $\mathfrak{m}(M)=2$. Choose a field $\mathbb{F}$ such that $H_{\mathfrak{m}}\left(M_{2} ; \mathbb{F}\right) \neq 0$. Then $H_{\mathfrak{m}}(\widetilde{M} ; \mathbb{F})$ is infinite-dimensional.

For any closed manifold homotopy equivalent to a connected sum $M=M_{1} \# M_{2}$ with $\pi_{1}\left(M_{1}\right)$ of order at least three and with $M_{2}$ simply-connected and not a homotopy sphere, it has been shown in $[26,27]$ by a different method ${ }^{1}$ that $\operatorname{dim} \iota_{*} H_{*}\left(\Omega_{0}^{T} M ; \mathbb{F}\right)$ grows even exponentially. It would therefore be interesting to find manifolds of nonfinite type that are not homotopy equivalent to a non-trivial connected sum. There are obstructions to split a manifold (up to homotopy type) into a non-trivial connected sum, see $[28, \S 7.4]$, but unfortunately these invariants are difficult to compute.

Question 1.4 How large is the class of closed manifolds of non-finite type that are not homotopy equivalent to a non-trivial connected sum?

\subsection{Applications}

Lower bounds for the rank of the homology of the sublevel sets $\Omega^{T} M$ are of interest because they classically lead, by Morse theory, to lower bounds for the number of geodesics of length $\leq T$ between non-conjugate points. Somewhat less classically, they also lead to lower bounds for the topological entropy of geodesic flows. Moreover, adding the tool of Floer homology, one gets lower bounds for the number of Reeb chords and for the topological entropy of Reeb flows on spherizations.

Before stating our two corollaries, we briefly recall what Reeb flows on spherizations are. Details can be found in the introduction of [8].

Reeb flows on spherizations. Consider a closed manifold $M$. The positive real numbers $\mathbb{R}_{+}$freely act on the cotangent bundle $T^{*} M$ by $r(q, p)=(q, r p)$. While the canonical 1-form $\lambda=p d q$ on $T^{*} M$ does not descend to the quotient $S^{*} M:=T^{*} M / \mathbb{R}_{+}$, its kernel does and defines a contact structure $\xi$ on $S^{*} M$. We call the contact manifold $\left(S^{*} M, \xi\right)$ the spherization of $M$. This contact manifold is co-orientable. The choice of a nowhere vanishing 1-form $\alpha$ on $S^{*} M$ with $\operatorname{ker} \alpha=\xi$ (called a contact form) defines a vector field $R_{\alpha}$ (the Reeb vector field of $\alpha$ ) by the two conditions $d \alpha\left(R_{\alpha}, \cdot\right)=0, \alpha\left(R_{\alpha}\right)=1$. Its flow $\varphi_{\alpha}^{t}$ is called the Reeb flow of $\alpha$.

To give a more concrete description of the manifold $\left(S^{*} M, \xi\right)$ and the flows $\varphi_{\alpha}^{t}$, consider a smooth hypersurface $\Sigma$ in $T^{*} M$ which is fiberwise starshaped with respect to the zero-section: For every $q \in M$ the set $\Sigma_{q}:=\Sigma \cap T_{q}^{*} M$ bounds a set in $T_{q}^{*} M$

\footnotetext{
${ }^{1}$ Paternain and Petean consider a suitable finite simply-connected subcomplex $K \subset \widetilde{M}$ and prove exponential homological growth of the based loop space of $K$ by showing exponential growth of the cohomology of the free loop space of $K$, which is isomorphic to the Hochschild homology of the singular cochain complex of $K$.
} 
that is strictly starshaped with respect to the origin of $T_{q}^{*} M$. The hyperplane field $\xi_{\Sigma}:=\operatorname{ker}\left(\left.\lambda\right|_{\Sigma}\right)$ is a contact structure on $\Sigma$, and the contact manifolds $\left(S^{*} M, \xi\right)$ and $\left(\Sigma, \xi_{\Sigma}\right)$ are isomorphic.

Let $\varphi_{\Sigma}^{t}$ be the Reeb flow on $\Sigma$ defined by the contact form $\left.\lambda\right|_{\Sigma}$. The set of Reeb flows on $\left(S^{*} M, \xi\right)$ can be identified with the Reeb flows $\varphi_{\Sigma}^{t}$ on the set of fiberwise starshaped hypersurfaces $\Sigma$ in $T^{*} M$. The flows $\varphi_{\Sigma}^{t}$ are restrictions of Hamiltonian flows: Consider a Hamiltonian function $H: T^{*} M \rightarrow \mathbb{R}$ such that $\Sigma=H^{-1}$ (1) is a regular energy surface and such that $H$ is fiberwise homogeneous of degree one near $\Sigma$. For the Hamiltonian flow $\varphi_{H}^{t}$ we then have $\left.\varphi_{H}^{t}\right|_{\Sigma}=\varphi_{\Sigma}^{t}$. It follows that geodesic flows and Finsler flows (up to the time change $t \mapsto 2 t$ ) are examples of Reeb flows on spherizations. Indeed, for geodesic flows the $\Sigma_{q}$ are ellipsoids, and for (symmetric) Finsler flows the $\Sigma_{q}$ are (symmetric and) convex. The flows $\varphi_{\Sigma}^{t}$ for varying $\Sigma$ are very different, in general, as is already clear from looking at geodesic flows on a sphere. In this paper we give uniform lower bounds for the growth of Reeb chords and for the complexity of all these flows on $\left(S^{*} M, \xi\right)$ for manifolds $M$ as in Theorem 1.1.

Growth of Reeb chords between two fibers. We say that $p, q \in M$ are non-conjugate points of the Reeb flow $\varphi_{\alpha}$ if $\bigcup_{t>0} \varphi_{\alpha}^{t}\left(S_{p}^{*} M\right)$ is transverse to $S_{q}^{*} M$. Given $p \in M$, the set of $q \in M$ that are non-conjugate to $p$ has full measure in $M$ by Sard's theorem. This notion of being non-conjugate generalizes the one in Riemannian geometry (defined in terms of Jacobi fields).

For $p, q \in M$ denote by $\mathcal{P}_{p q}$ the space of continuous paths in $S^{*} M$ from $S_{p}^{*} M$ to $S_{q}^{*} M$, and by $\Omega_{p q} M$ the space of continuous paths in $M$ from $p$ to $q$. We shall assume throughout that $\operatorname{dim} M \geq 3$, since otherwise $M$ is of finite type. The fibers $S_{q}^{*} M$ of the projection $p r: S^{*} M \rightarrow M$ are then simply connected, and so $p r$ induces a bijection on the components of $\mathcal{P}_{p q}$ and $\Omega_{p q} M$. The space $\Omega_{p q} M$ is homotopy equivalent to $\Omega_{p} M:=\Omega_{p, p} M$, whose components are parametrized by the elements of the fundamental group $\pi_{1}(M, p)$.

Corollary 1.1 Assume that $M$ is not of finite type, has virtually polycyclic fundamental group, and that there is a field $\mathbb{F}$ such that $H_{\mathfrak{m}}(\widetilde{M} ; \mathbb{F})$ is infinite-dimensional. Let $\left(S^{*} M, \xi\right)$ be the spherization of $M$. Then for any Reeb flow $\varphi_{\alpha}$ on $\left(S^{*} M, \xi\right)$, any pair of non-conjugate points $p, q \in M$ and every component $\mathcal{C}$ of $\Omega_{p q} M$, the number of Reeb chords from $S_{p}^{*} M$ to $S_{q}^{*} M$ that belong to $\mathcal{C}$ grows in time at least like $e^{\sqrt{T}}$.

Remark 1.5 (i) For the special case of geodesic flows, the time parameter equals the length run through. Hence the corollary translates to assertion (i) of the abstract.

To illustrate the corollary, we choose a Riemannian metric on $M$ and a point $p \in M$. Let $C(p)$ be the cut locus of $p$. The subset $M \backslash C(p)$ is diffeomorphic to an open ball [23] and has full measure in $M$. For every $q \in M \backslash C(p)$ there is a unique shortest geodesic $c_{q}$ from $p$ to $q$. Call a path $\gamma \in \Omega_{p q} M$ contractible if $c_{q}^{-1} \circ \gamma$ is contractible in $\Omega_{p} M$. The set $U_{p}$ of points in $M \backslash C(p)$ that are not conjugate to $p$ is also of full measure in $M$. Under the hypothesis of Corollary 1.1, for every $q \in U_{p}$ the number of contractible geodesics from $p$ to $q$ of length $\leq T$ grows at least like $e^{\sqrt{T}}$.

(ii) Virtually polycyclic groups are either virtually nilpotent or have exponential growth [34]. If the fundamental group $\pi_{1}(M)$ of a closed manifold $M$ has expo- 
nential growth, then the number of Reeb chords from $S_{p}^{*} M$ to $S_{q}^{*} M$ grows exponentially in time for any, possibly conjugate, pair of points $p, q$ (see [19, Corollary 1] for Reeb flows). Indeed, one finds one Reeb chord for each element of $\pi_{1}(M)$. In Corollary 1.1, however, we find " $e^{\sqrt{T}}$ many" Reeb chords for each element of $\pi_{1}(M)$.

If one is only interested in the growth of Reeb chords from $S_{p}^{*} M$ to $S_{q}^{*} M$, without specifying the component $\mathcal{C}$, then Corollary 1.1 is interesting only for virtually nilpotent fundamental groups, which by Gromov's theorem from [12] are exactly those fundamental groups that grow polynomially. Indeed, it is believed that every finitely presented group that grows more than polynomially grows exponentially [9, Conjecture 11.3], and even for finitely generated groups of intermediate growth it is believed that they must grow at least like $e^{\sqrt{T}}$, cf. [10]. (iii) Let $\mu(\gamma)$ be the Conley-Zehnder index of a non-degenerate Reeb chord $\gamma$ on $\left(S^{*} M ; \alpha\right)$, normalized such that for geodesic flows $\mu(\gamma)$ is the Morse index of the non-degenerate geodesic $\gamma$ (i.e. the number of conjugate points, counted with multiplicities, along $\gamma$ ). In the situation of Corollary 1.1, Remark 1.2 (ii) shows that for every component of $\Omega_{p q} M$ the number of Reeb chords from $S_{p}^{*} M$ to $S_{q}^{*} M$ of index $\mu(\gamma)=k$ is infinite if $k=\mathfrak{m}-1$ and is at least the number $q(n)$ of partitions of $n$ into distinct parts if $k=(\mathfrak{m}-1) n$ and $n \geq 2$.

Intermediate volume growth. Consider a smooth diffeomorphism $\varphi$ of a closed manifold $X$. Denote by $\mathcal{S}$ the set of smooth compact submanifolds of $X$. Fix a Riemannian metric $\rho$ on $X$, and denote by $\operatorname{Vol}(\sigma)$ the $j$-dimensional volume of a $j$-dimensional submanifold $\sigma \in \mathcal{S}$ computed with respect to the measure on $\sigma$ induced by $\rho$. For $a \in(0,1]$ define the intermediate volume growth of $\sigma \in \mathcal{S}$ by

$$
\operatorname{vol}^{a}(\sigma ; \varphi)=\liminf _{n \rightarrow \infty} \frac{\log \operatorname{Vol}\left(\varphi^{n}(\sigma)\right)}{n^{a}} \in[0, \infty],
$$

and define the intermediate volume growth of $\varphi$ by

$$
\operatorname{vol}^{a}(\varphi)=\sup _{\sigma \in \mathcal{S}} \operatorname{vol}^{a}(\sigma ; \varphi) \in[0, \infty] .
$$

Notice that these invariants do not depend on the choice of $\rho$. Finally define the volume growth exponent of $\varphi$ by

$$
\exp _{\mathrm{vol}}(\varphi):=\inf \left\{a \mid \operatorname{vol}^{a}(\varphi)<\infty\right\} .
$$

Thus $\exp _{\mathrm{vol}}(\varphi)$ is "the largest $a \in[0,1]$ such that some submanifold grows under $\varphi$ like $e^{n^{a}}$." Since $\operatorname{vol}^{1}(\varphi) \leq(\operatorname{dim} X) \max _{x \in X}\|D \varphi(x)\|<\infty$ we have $\exp _{\text {vol }}(\varphi) \in$ $[0,1]$. The intermediate volume growth and the volume growth exponent of a smooth flow $\varphi^{t}$ on $X$ are defined as $\operatorname{vol}^{a}\left(\varphi^{1}\right)$ and $\exp _{\mathrm{vol}}\left(\varphi^{1}\right)$.

Remark By a celebrated result of Yomdin [35] and Newhouse [22], the volume growth $\operatorname{vol}^{1}(\varphi)$ agrees with the topological entropy $h_{\text {top }}(\varphi)$. Proceeding as above define for $a \in(0,1]$ the intermediate topological entropy $h_{\text {top }}^{a}(\varphi)$. We unfortunately do not know whether $\operatorname{vol}^{a}(\varphi)=h_{\text {top }}^{a}(\varphi)$ or at least that $\operatorname{vol}^{a}(\varphi) \leq h_{\text {top }}^{a}(\varphi)$ also for $a \in(0,1)$. 
Uniform lower bounds for the volume growth or the topological entropy of geodesic flows were found in [5,24,25,27], and these results were generalized to Reeb flows in [19] and, on a polynomial scale, in [8]. Results in [8,19] show that the volume growth exponent $\exp _{\mathrm{vol}}\left(\varphi_{\alpha}\right)$ is bounded from below by the maximum of the growth exponent of the function $T \mapsto \operatorname{rank} \iota_{*}^{T} H_{*}\left(\Omega_{0}^{T} M\right)$ and the growth exponent of the growth function of the fundamental group $\pi_{1}(M)$. In particular, for manifolds with fundamental group of exponential growth it is shown in [19] that $\operatorname{vol}^{1}\left(\varphi_{\alpha}\right)>0$ for any Reeb flow $\varphi_{\alpha}$ on $S^{*} M$. Since virtually polycyclic groups are either virtually nilpotent or have exponential growth, we therefore restrict ourselves now to manifolds with virtually nilpotent fundamental group. Since all other fundamental groups of closed manifolds are believed to have exponential growth, this is a minor hypothesis.

Corollary 1.2 Assume that $M$ is not of finite type, has virtually nilpotent fundamental group, and that there is a field $\mathbb{F}$ such that $H_{\mathfrak{m}}(\widetilde{M} ; \mathbb{F})$ is infinite-dimensional. Let $\left(S^{*} M, \xi\right)$ be the spherization of $M$. Then

$$
\operatorname{vol}^{1 / 2}\left(S_{p}^{*} M ; \varphi_{\alpha}\right)>0
$$

for every fiber $S_{p}^{*} M$ of $S^{*} M$ and every Reeb flow $\varphi_{\alpha}$ on $\left(S^{*} M\right.$, $\left.\xi\right)$. In particular, $\operatorname{vol}^{1 / 2}\left(\varphi_{\alpha}\right)>0$ and $\exp _{\mathrm{vol}}\left(\varphi_{\alpha}\right) \geq 1 / 2$ for every Reeb flow $\varphi_{\alpha}$ on $\left(S^{*} M, \xi\right)$.

The method. We end this introduction by comparing our approach to previous approaches. As mentioned earlier, lower bounds for the homology of the sublevel sets $\Omega^{T} M$ follow easily from the growth of the fundamental group $\pi_{1}(M)$, since its growth is the growth of $H_{0}\left(\Omega^{T} M\right)$. If $\pi_{1}(M)$ is finite, Gromov found a way to bound the rank of $H_{*}\left(\Omega^{T} M\right)$ from below by the rank of $\oplus_{i=0}^{c T} H_{i}(\Omega M)$, where $c$ is a constant depending only on the Riemannian metric. His ingenious argument is purely geometric, see $[11,13,24]$, and uses the fact that $\widetilde{M}$ is compact. In [26,27] Paternain-Petean generalized Gromov's construction to manifolds with infinite fundamental group, by mapping simply connected complexes $K$ with rich loop space homology into $\widetilde{M}$. This method gives good lower bounds for the rank of $H_{*}\left(\Omega^{T} M\right)$ if one can find such complexes and mappings $f: K \rightarrow \widetilde{M}$ for which the homology of $\Omega f(\Omega K)$ still has large rank. This works well for many manifolds, e.g. for most connected sums, for manifolds of finite type with $\pi_{*}(\Omega M) \otimes \mathbb{Q}$ infinite-dimensional, and in small dimensions, see [26,27].

To fix the ideas, take as $K$ the wedge sum $S^{n} \vee S^{n}$ of two $n$-spheres. The homology algebra $H_{*}(\Omega K)$ is the free associative algebra generated by the two generators $x_{1}, x_{2}$ of $H_{n-1}(\Omega K) \cong H_{n}(K)$, see [4, III. 1. B]. If one can find continuous maps $\iota: K \rightarrow M$ and $r: M \rightarrow K$ such that $r \circ \iota$ is homotopic to the identity of $K$, then $\Omega \iota: \Omega K \rightarrow \Omega M$ induces an injection in homology. One then concludes that the rank of $\iota_{*}^{T} H_{*}\left(\Omega^{T} M\right)$ grows exponentially. This method was successfully used in dimension $\leq 5$ in [27].

For general manifolds of non-finite type, however, we were not even able to prove linear growth of the rank of $H_{*}\left(\Omega^{T} M\right)$ by this method. Citing G. Paternain, "it is as if one has so much topology that it becomes unmanageable." 


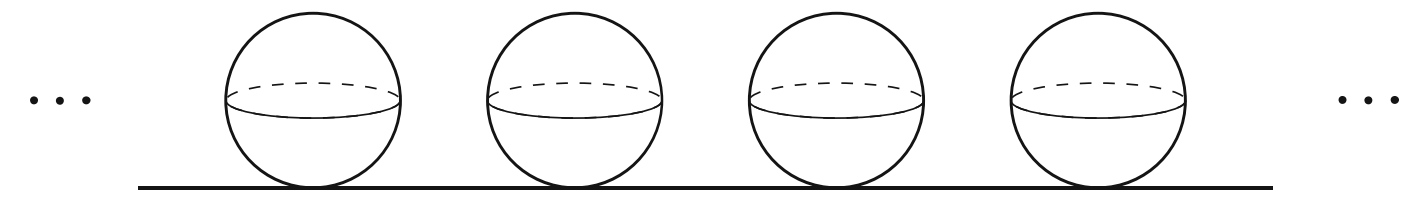

Our strategy to prove lower bounds for the rank of $H_{*}\left(\Omega^{T} M\right)$ for manifolds of nonfinite type is to find an infinite wedge sum $\vee_{i \in \mathbb{Z}} S_{i}^{n}$ of spheres in $M$ as in the figure and to prove that the ordered words $x_{i_{1}} x_{i_{2}} \cdots x_{i_{k}}, i_{1}<i_{2}<\cdots<i_{k}$, are still independent in $H_{*}(\Omega M ; \mathbb{F})$. The proof is neither geometric nor topological, but algebraic. The main point is that we use the Hopf algebra structure of $H_{*}(\Omega \widetilde{M} ; \mathbb{F})$ over the group ring $\mathbb{F}\left[\pi_{1}(M)\right]$, and prove a Poincaré-Birkhoff-Witt type theorem for this algebra. These algebraic results (specifically Theorems 2.1 and 3.1 and Proposition 4.1) are the main findings of this paper. They cover Sects. 2-4. Theorem 1.1, which is proven in Sect. 5, follows readily from these algebraic results, and Corollaries 1.1 and 1.2, which are proven in Sect. 7, follow from Theorem 1.1 by a standard continuation argument in Floer homology and by looking at the volume growth of the Lagrangian disc in $T_{p}^{*} M$ bounded by $\Sigma_{p}$.

\section{Healthy $G$-vector spaces}

Let $G$ be a group, $V$ a vector space over the field $\mathbb{F}$, and $\rho: G \rightarrow \operatorname{Aut}(V)$ a representation of $G$. Since the representation is fixed throughout our discussion, we abbreviate $g v=\rho(g) v$ for $g \in G$ and $v \in V$, and we consider the $G$-vector space $V$ as a module over the group ring $\mathbb{F} G$. A group $G$ is called polycyclic if there exists a subnormal series

$$
1=G_{0} \triangleleft G_{1} \triangleleft \cdots \triangleleft G_{n}=G
$$

such that all factors $G_{i} / G_{i-1}, 1 \leq i \leq n$ are cyclic. A group $G$ is called virtually polycyclic if $G$ has a polycyclic subgroup of finite index. Eleven characterisations of polycyclic groups are given in [34, Proposition 4.1]. In this section we prove the following result.

Theorem 2.1 Assume that $G$ is a virtually polycyclic group acting linearly on an infinite-dimensional vector space $V$ such that $V$ viewed as an $\mathbb{F} G$-module is finitely generated. Then there exists $v \in V$ and $g \in G$ such that the sequence of vectors $\left(g^{i} v\right)_{i \in \mathbb{Z}}$ is linearly independent.

Before embarking on the proof of the theorem we introduce some notation. For the following discussion it is irrelevant that $G$ is virtually polycyclic. Given $g \in G$ and $v \in V$ we denote the subvector space of $V$ spanned by the vectors $g^{i} v$ by

$$
W_{v}^{g}:=\left\langle g^{i} v \mid i \in \mathbb{Z}\right\rangle \subset V
$$

Definition 2.1 A vector $v \in V$ is healthy if there exists $g \in G$ such that $W_{v}^{g}$ is infinite-dimensional. A vector $v \in V$ which is not healthy is called sick. 
The reason why we are interested in finding healthy vectors is the following observation from linear algebra.

Lemma 2.1 If $g \in G$ and $v \in V$, then the following are equivalent.

(i) $W_{v}^{g}$ is infinite-dimensional.

(ii) The sequence of vectors $\left(g^{i} v\right)_{i \in \mathbb{Z}}$ is linearly independent.

Proof That (ii) implies (i) is clear. It remains to show that if (ii) does not hold, then $W_{v}^{g}$ is finite-dimensional. The case $v=0$ is trivial as well, so we assume that $v \neq 0$. Since then $g^{-i}\left(g^{i} v\right)=v \neq 0$, we conclude that $g^{i} v \neq 0$ for every $i \in \mathbb{Z}$. Hence, if (ii) does not hold, there exist $m<n \in \mathbb{Z}$ and scalars $a_{i} \in \mathbb{F}$ for $m \leq i \leq n-1$ such that

$$
g^{n} v=\sum_{i=m}^{n-1} a_{i} g^{i} v, \quad a_{m} \neq 0
$$

After applying $g^{-m}$ to (2.1) we can assume without loss of generality that $m=0$. Applying $g$ and $g^{-1}$ to (2.1) we obtain inductively that

$$
W_{v}^{g}=\left\langle g^{i} v \mid 0 \leq i \leq n-1\right\rangle \text {. }
$$

This shows that $W_{v}^{g}$ is finite-dimensional. The lemma follows.

If $v \neq 0$ is a sick vector, we can define in view of (2.2) the function $d_{v}: G \rightarrow \mathbb{N}$ by

$$
d_{v}(g):=\min \left\{n \in \mathbb{N} \mid W_{v}^{g}=\left\langle g^{i} v \mid 0 \leq i \leq n-1\right\rangle\right\}=\operatorname{dim} W_{v}^{g} .
$$

Definition 2.2 The $G$-vector space $V$ is called sick if all its vectors are sick. A $G$-vector space $V$ which is not sick is called healthy, i.e., $V$ contains a healthy vector.

Note that a healthy vector space still contains sick vectors. Indeed, the zero vector is always sick. Moreover, observe that the concept of a healthy $G$-vector space is only of interest if both the cardinality of the group and the dimension of the vector space are infinite, since otherwise $V$ is automatically sick.

Although we fix the representation $\rho$ throughout, we sometimes have to restrict $\rho$ to subgroups $H<G$. In this situation, we say that $V$ is $H$-healthy if the restriction of $\rho$ to $H$ is healthy.

Lemma 2.2 Assume that $H<G$ is a subgroup of finite index. Then $V$ is $G$-healthy if and only if $V$ is $H$-healthy.

Proof The implication from $H$-healthy to $G$-healthy is obvious. We now assume that $V$ is $G$-healthy and show that $V$ is $H$-healthy as well. Since $V$ is $G$-healthy, there exists a vector $v \in V$ and a group element $g \in G$ such that $W_{v}^{g}$ is infinite-dimensional. Denote the right coset $g H$ in $G / H$ by $[g]$. 
We first consider the special case where the subgroup $H$ is normal. Then $G / H$ is a group, and its order $n:=|G / H|$ is finite by assumption. Hence $[g]^{n}=i d \in G / H$, or equivalently $h:=g^{n} \in H$. By Lemma 2.1 we conclude from the fact that $W_{v}^{g}$ is infinite-dimensional that $W_{v}^{h}$ is infinite-dimensional as well. This proves that $V$ is $H$-healthy in the special case that $H \triangleleft G$ is normal.

In the general case where $H<G$ is not necessarily normal, we consider the normal core of $H$ in $G$ defined by

$$
\operatorname{Core}(H):=\bigcap_{g \in G} g^{-1} H g \text {. }
$$

Note that Core $(H)$ is a subgroup of $H$ which is normal in $G$. It is actually the biggest normal subgroup of $G$ contained in $H$. Moreover, it still has finite index in $H$, see for instance [29, Theorem 3.3.5]. In view of what we already proved, we therefore conclude that $V$ is Core $(H)$-healthy. Since Core $(H)<H$ it follows that $V$ is $H$ healthy as well. This finishes the proof of the lemma.

If $H<G$ is a subgroup and $v \in V$, we abbreviate by

$$
W_{v}^{H}=\langle H v\rangle
$$

the subspace of $V$ spanned by the $H$-orbit of the vector $v$. The next lemma is our main tool to give an inductive proof of Theorem 2.1.

Lemma 2.3 Assume that $H \triangleleft G$ is a normal subgroup such that $G / H$ is cyclic. Suppose further that $v \in V$ is sick and $W_{v}^{H}$ is finite-dimensional. Then $W_{v}^{G}$ is finitedimensional as well.

Proof Abbreviate $m=\operatorname{dim} W_{v}^{H}$. Then there exist $\xi_{1}, \ldots, \xi_{m}$ in the group $\operatorname{ring} \mathbb{F} H$ such that

$$
W_{v}^{H}=\left\langle\xi_{i} v \mid 1 \leq i \leq m\right\rangle
$$

Choose $g \in G$ such that $[g] \in G / H$ is a generator. Assume first that $G / H \cong \mathbb{Z}_{n}$ is finite. Then

$$
W_{v}^{G}=\left\langle g^{j} \xi_{i} v \mid 1 \leq i \leq m, 0 \leq j<n\right\rangle
$$

Hence $\operatorname{dim} W_{v}^{g} \leq n m$ is finite. Assume now that $G / H \cong \mathbb{Z}$ is infinite. The case that $v=0$ is trivial. We therefore assume that $v \neq 0$. Since $v$ is sick by assumption, we have the function $d_{v}: G \rightarrow \mathbb{N}$ from (2.3), and we abbreviate $n=d_{v}(g)$. Our aim is to show that

$$
W_{v}^{G}=\left\langle g^{j} \xi_{i} v \mid 1 \leq i \leq m, 0 \leq j<n\right\rangle
$$


For this purpose we abbreviate the right hand side by

$$
X:=\left\langle g^{j} \xi_{i} v \mid 1 \leq i \leq m, 0 \leq j<n\right\rangle
$$

That $X \subset W_{v}^{G}$ is clear. We have to check the other inclusion, namely that for every $\eta \in \mathbb{F} G$ it holds that

$$
\eta v \in X
$$

Since the right coset $[g]$ is a generator of $G / H \cong \mathbb{Z}$, there exist $\eta_{j} \in \mathbb{F} H$ with $\eta_{j} \neq 0$ for only finitely many $j \in \mathbb{Z}$ such that

$$
\eta=\sum_{j \in \mathbb{Z}} \eta_{j} g^{j}
$$

In view of the definition of $n=d_{v}(g)$, there exists $\zeta \in \mathbb{F} G$ of the form

$$
\zeta=\sum_{j=0}^{n-1} \zeta_{j} g^{j}, \quad \zeta_{j} \in \mathbb{F} H
$$

such that

$$
\eta v=\zeta v
$$

Since $H$ is normal in $G$, there exist $\zeta_{j}^{\prime} \in \mathbb{F} H$ for $0 \leq j<n$ such that

$$
\zeta=\sum_{j=0}^{n-1} g^{j} \zeta_{j}^{\prime}
$$

In view of (2.4) we conclude that for $0 \leq j<n$ we have

$$
\zeta_{j}^{\prime} v \in\left\langle\xi_{i} v \mid 1 \leq i \leq m\right\rangle
$$

Therefore $\eta v=\zeta v \in X$. This proves (2.5). We have shown that

$$
\operatorname{dim} W_{v}^{G} \leq n m=d_{v}(g) \cdot \operatorname{dim} W_{v}^{H},
$$

and therefore $W_{v}^{G}$ is finite-dimensional. The proof of the lemma is complete.

We are now in position to prove the main result of this section.

Proof of Theorem 2.1 In view of Lemma 2.1 it suffices to show that $V$ is healthy. We argue by contradiction and assume that $V$ is sick. By assumption $G$ is virtually polycyclic, hence contains a polycyclic subgroup $H$ of finite index. By Lemma 2.2 
it follows that $V$ is $H$-sick as well. Since $H$ is polycyclic, we conclude by applying Lemma 2.3 inductively that $W_{v}^{H}$ is finite-dimensional for every $v \in V$. Because $H$ has finite index in $G$ it follows that $W_{v}^{G}$ is finite-dimensional for every $v \in V$ as well. By assumption $V$ is a finitely generated $\mathbb{F} G$-module. Therefore we deduce that $V$ is finite-dimensional, contradicting the assumption of the theorem. The proof is complete.

\section{Filtered Hopf algebras}

In this section we consider Hopf algebras $V$ over a group ring $\mathbb{F} G$, endowed with a filtration $V^{r}, r>0$, and exhibit a property of $V$ that guarantees that $\operatorname{dim} V^{r}$ grows at least like $e^{\sqrt{r}}$.

\subsection{Hopf algebras over $\mathbb{F} G$}

Let $G$ be a group, $\mathbb{F}$ a field and $\mathbb{F} G$ the group ring. We first explain the notion of a Hopf algebra over $\mathbb{F} G$. This might be not completely standard, since usually Hopf algebras are defined over rings which are commutative, a requirement that our group ring in general does not fulfill. However, the feature which distinguishes a group ring from other non-commutative rings is that if $V$ and $W$ are two left modules over $\mathbb{F} G$, then we can still define on their tensor product $V \otimes W=V \otimes_{\mathbb{F}} W$ the structure of a left $\mathbb{F} G$-module by using the tensor of the two representations: $g(v \otimes w):=(g v) \otimes(g w)$ for $v \in V, w \in W$ and $g \in G$. A product is then an $\mathbb{F} G$-linear map $\mu: V \otimes V \rightarrow V$, or equivalently an $\mathbb{F}$-bilinear map $\mu: V \times V \rightarrow V$ satisfying $\mu(g v, g w)=g \mu(v, w)$ for $g \in G$ and $v, w \in V$. Dually, a coproduct is then an $\mathbb{F} G$-linear map $\Delta: V \rightarrow$ $V \otimes V$. To avoid terrible headaches we assume in addition that our product is always associative, although this requirement is probably not necessary for the results of this section. We abbreviate the product by $v w=\mu(v, w)$. If $V$ in addition is graded, i.e. $V=\bigoplus_{i=0}^{\infty} V_{i}$, where each $V_{i}$ is an $\mathbb{F} G$-submodule of $V$, then we grade the tensor product by $(V \otimes V)_{k}=\bigoplus_{i+j=k} V_{i} \otimes V_{j}$ and require in addition that the product and coproduct preserve the grading. The product endows the tensor product $V \otimes V$ again with a product which is defined on homogeneous elements by the Koszul sign convention

$$
(v \otimes w)(x \otimes y)=(-1)^{\operatorname{deg}(w) \operatorname{deg}(x)} v x \otimes w y
$$

where $\operatorname{deg}(v)$ denotes the degree of a homogeneous element $v$. Given a left module $V$ over $\mathbb{F} G$ and a product $\mu$ and coproduct $\Delta$ as above, we call the triple $(V, \mu, \Delta)$ a bialgebra over $\mathbb{F} G$ if $\mu$ and $\Delta$ are compatible in the sense that $\Delta: V \rightarrow V \otimes V$ is a homomorphism of algebras. The bialgebra $(V, \mu, \Delta)$ is called connected if $V_{0}=\mathbb{F}$ is one-dimensional and if $1 \in \mathbb{F}$ is also the unit for the multiplication $\mu$.

Definition 3.1 A connected graded bialgebra $(V, \mu, \Delta)$ over $\mathbb{F} G$ is called a Hopf algebra over $\mathbb{F} G$ if for every homogeneous element $v$ of positive degree $\operatorname{deg}(v)$ the 
coproduct satisfies

$$
\Delta v=1 \otimes v+v \otimes 1+\sum v_{i} \otimes v_{i}^{\prime}
$$

with $v_{i}$ and $v_{i}^{\prime}$ of positive degree. A vector $v \in V$ is primitive if $\Delta v=1 \otimes v+v \otimes 1$.

\subsection{Filtrations}

In order to endow our Hopf algebra with a filtration we shall suppose that both the vector space $V$ and the group ring $\mathbb{F} G$ are filtered. More precisely, we assume that $V$ can be exhausted by a sequence of finite-dimensional vector spaces, i.e., for every real number $r>0$ there exists a finite-dimensional subspace $V^{r} \subset V$ such that $V^{r} \subset V^{s}$ for $r \leq s$ and $V=\bigcup_{r>0} V^{r}$. Define the value of $v \in V$ by

$$
|v|:=\min \left\{r \mid v \in V^{r}\right\}
$$

Notice that for scalars $a_{i} \in \mathbb{F}$ and vectors $v_{i} \in V$ we have

$$
\left|a_{1} v_{1}+\cdots+a_{n} v_{n}\right| \leq \max \left\{\left|v_{i}\right|\right\}
$$

Dear reader, please do not confuse the value $|v|$ of $v$ and its degree $\operatorname{deg}(v)$, in case $v$ is homogeneous. They are not related to each other. Also note that the grading is indicated by a subscript while the filtration degree is indicated by a superscript. To get a filtration on the group ring as well, suppose that the group $G$ is endowed with a length function, namely a function $L: G \rightarrow \mathbb{R}_{\geq 0}$ satisfying

$$
L(g)=L\left(g^{-1}\right), \quad L(g h) \leq L(g)+L(h), \quad g, h \in G .
$$

In the following we abbreviate $|g|=L(g)$. Via the length function we can define a filtration on the group ring: For $r \geq 0$ we define $\mathbb{F} G^{r}$ to be the subvector space of $\mathbb{F} G$ consisting of finite sums $\xi=\sum_{g \in G} \xi_{g} g$ satisfying $\xi_{g}=0$ whenever $|g|>r$.

Definition 3.2 The Hopf algebra $(V, \mu, \Delta)$ over $\mathbb{F} G$ is called filtered if the vector space $V$ is endowed with a filtration such that for $v, w \in V$ and $g \in G$ it holds that

$$
|v w| \leq|v|+|w|, \quad|g v| \leq|g|+|v|
$$

We next introduce a condition on filtered Hopf algebras which will guarantee nontrivial lower bounds for the growth of $\operatorname{dim} V^{r}$.

Definition 3.3 Assume that $(V, \mu, \Delta)$ is a filtered Hopf algebra over $\mathbb{F} G$. A primitive sequence is a sequence $\left(v_{i}\right)_{i \in \mathbb{N}}$ satisfying the following conditions.

(i) The vectors $v_{i}, i \in \mathbb{N}$, are linearly independent, primitive, and of equal positive degree.

(ii) There exists a constant $c>0$ such that $\left|v_{i}\right| \leq c i$. 
Remark The notion of a primitive sequence does not involve the action of the group $G$. However, we shall see later that the action of $G$ is useful to construct primitive sequences.

The following result, whose proof is deferred to the next section, will be an important ingredient in the proof of the subsequent Theorem 3.1, which is the main result of this section.

Proposition 3.1 Let $(V, \mu, \Delta)$ be a Hopf algebra over $\mathbb{F}$, and let $v_{1}, \ldots, v_{N}$ be linearly independent and primitive vectors in $V$ of equal degree. For $I=\left\{i_{1}, i_{2}, \ldots, i_{\ell}\right\}$ with $1 \leq i_{1}<i_{2}<i_{\ell} \leq N$ write $v_{I}=v_{i_{1}} v_{i_{2}} \cdots v_{i_{\ell}}$. Then the vectors $v_{I}$, $I \subset\{1, \ldots, N\}$, are linearly independent.

Theorem 3.1 Assume that $(V, \mu, \Delta)$ is a filtered Hopf algebra over $\mathbb{F} G$ which admits a primitive sequence $\left(v_{i}\right)_{i \in \mathbb{N}}$. Then the function $r \mapsto \operatorname{dim} V^{r}$ grows at least like $e^{\sqrt{r}}$.

Proof Let $I=\left\{i_{1}, \ldots, i_{\ell}\right\} \subset \mathbb{N}$ be a finite subset of distinct numbers which we totally order by $i_{1}<i_{2}<\cdots<i_{\ell}$, and abbreviate

$$
v_{I}=v_{i_{1}} v_{i_{2}} \cdots v_{i_{\ell}} \in V
$$

Let $m$ be the common degree of the vectors $v_{i}$. In view of property (i) of a primitive sequence, Proposition 3.1 shows that the vectors $v_{I}, I \subset \mathbb{N}$, are linearly independent. We can assume without loss of generality that the constant $c$ in property (ii) of a primitive sequence is 1 . Then we have

$$
\left|v_{I}\right| \leq \sum_{j=1}^{\ell}\left|v_{i_{j}}\right| \leq \sum_{j=1}^{\ell} i_{j}
$$

For $k \in \mathbb{N}$ denote by $q(k)$ the number of partitions of $k$ into distinct parts. We have shown that

$$
\operatorname{dim} V^{n} \geq q(1)+q(2)+\cdots+q(n) \geq q(n) .
$$

By Euler's theorem, the number of partitions of $n$ into distinct parts coincides with the number of its partitions into odd parts, see for example [3, Corollary 1.2]. The asymptotics of this sequence coincides up to a constant with the asymptotics of the partition function (see for example [21, Chapter 16]), which grows like $e^{C \sqrt{n}}$ for a positive constant $C$ according to a theorem of Hardy and Ramanujan, see for example [21, Chapter 15].

Combining Theorems 2.1 and 3.1 we obtain the following result.

Corollary 3.1 Assume that $(V, \mu, \Delta)$ is a filtered Hopf algebra over $\mathbb{F} G$ where $G$ is a virtually polycyclic group. Assume further that $\oplus_{i<m} V_{i}$ is finite-dimensional and that $V_{m}$ is infinite-dimensional but finitely generated as an $\mathbb{F} G$-module. Then the function $r \mapsto \operatorname{dim} V^{r}$ grows at least like $e^{\sqrt{r}}$. 
Proof By Theorem 2.1 there exists $v \in V_{m}$ and $g \in G$ such that the vectors $v_{i}:=g^{i} v$, $i \in \mathbb{N}$, are linearly independent. The vectors $v_{i}$ have equal degree $m$. Using the defining properties of a filtration, we estimate

$$
\left|v_{i}\right|=\left|g^{i} v\right| \leq\left|g^{i}\right|+|v| \leq i|g|+|v|
$$

With $c:=|g|+|v|$ we thus have $\left|v_{i}\right| \leq c i$. The sequence $\left(v_{i}\right)_{i \in \mathbb{N}}$ therefore meets all the properties of a primitive sequence, except that the $v_{i}$ may fail to be primitive. To correct this, we consider the linear map $A:\left\langle v_{1}, v_{2}, \ldots\right\rangle \rightarrow V_{m} \otimes V_{m}$ given by

$$
A(v)=\Delta v-1 \otimes v-v \otimes 1
$$

Since the vector space $\oplus_{j<m} V_{j}$ is finite-dimensional, the subvector space of $V_{m} \otimes V_{m}$ spanned by the elements $u_{i} \otimes u_{i}^{\prime}$ with $u_{i}, u_{i}^{\prime} \in \oplus_{j<m} V_{j}$ is finite-dimensional. Since $A$ takes values in this finite-dimensional vector space, $k:=\operatorname{rank} A$, which equals the dimension of $\left\langle v_{1}, v_{2}, \ldots\right\rangle / \operatorname{ker} A$, is finite. In particular, $\operatorname{ker} A$ in infinite-dimensional. We shall construct by induction a sequence $w_{1}, w_{2}, \ldots$ of linearly independent elements in $\operatorname{ker} A$ such that $\left|w_{i}\right| \leq c(k+1)$. The sequence $\left(w_{i}\right)_{i \in \mathbb{N}}$ is then a primitive sequence in $V$, and the corollary follows in view of Theorem 3.1.

The restriction of the map $A$ to $\left\langle v_{1}, v_{2}, \ldots, v_{k+1}\right\rangle$ has a non-trivial kernel. Let $w_{1}:=a_{1} v_{1}+\cdots+a_{k+1} v_{k+1}$ be a non-trivial element in this kernel. Then by (3.2), $\left|w_{1}\right| \leq c(k+1)$. Next, the restriction of $A$ to $\left\langle v_{k+2}, v_{k+3}, \ldots, v_{2(k+1)}\right\rangle$ has a nontrivial kernel. Let $w_{2}:=a_{k+2} v_{k+2}+\cdots+a_{2(k+1)} v_{2(k+1)}$ be a non-trivial element in this kernel. Then by (3.2), $\left|w_{2}\right| \leq 2 c(k+1)$, and $w_{1}, w_{2}$ are linearly independent because $v_{1}, \ldots, v_{2(k+1)}$ are linearly independent. Proceeding in this way we construct linearly independent vectors $w_{1}, w_{2}, \ldots$ such that $\left|w_{i}\right| \leq i c(k+1)$.

\section{A quantum Poincaré-Birkhoff-Witt theorem}

Consider a graded bialgebra $(V, \mu, \Delta)$ over the field $\mathbb{F}$ which is connected (i.e., $V_{0}=\mathbb{F}$ is one-dimensional) and is such that $1 \in \mathbb{F}$ also serves as the unit for the multiplication $\mu$. (The group $G$ plays no role in this section.) We again assume that $\mu$ is associative, and write $v w=\mu(v, w)$. Also recall that $v \in V$ is primitive if $\Delta(v)=1 \otimes v+v \otimes 1$. Suppose that for $N \in \mathbb{N}$ we are given linearly independent and primitive elements $v_{1}, \ldots, v_{N} \in V_{m}$ of equal positive degree $m \in \mathbb{N}$. Abbreviate $\mathbb{N}_{N}=\{1, \ldots, N\}$. We order $I \subset \mathbb{N}_{N}$ using the canonical order of $\mathbb{N}_{N}$, namely we write $I=\left\{i_{1}, \ldots, i_{\ell}\right\}$ satisfying $i_{1}<i_{2}<\cdots<i_{\ell}$. We then abbreviate $v_{I}=v_{i_{1}} v_{i_{2}} \cdots v_{i_{\ell}}$, where we use the convention that $v_{\emptyset}=1$. The following proposition reminiscent of the Poincaré-Birkhoff-Witt theorem seems to be known to people working in quantum group theory, cf. [31, Theorem 1.5(b)]. However, since the Hopf algebras arising in the theory of quantum groups are usually not graded, we provide a proof for the readers convenience.

Proposition 4.1 Assume that $v_{1}, \ldots, v_{N}$ are linearly independent and primitive vectors in $V$. Then the vectors $v_{I}, I \subset \mathbb{N}_{N}$, are linearly independent. 
Proof The crucial ingredient in the proof is the computation of the coproduct of the elements $v_{I}$. To determine the signs in this formula, we use the following convention. Given a subset $I$ of $\{1, \ldots, N\}$ we order $I=\left\{i_{1}, \ldots, i_{\ell}\right\}$ and its complement $I^{c}=\left\{j_{1}, \ldots, j_{N-\ell}\right\}$. This determines a permutation $(1, \ldots, N) \mapsto$ $\left(i_{1}, \ldots, i_{\ell}, j_{1}, \ldots, j_{N-\ell}\right)$. We denote by $\sigma(I)$ the signum of this permutation.

Lemma 4.1 The coproduct of $v_{\mathbb{N}_{N}}$ is given by

$$
\Delta\left(v_{\mathbb{N}_{N}}\right)=\sum_{I \subset \mathbb{N}_{N}} \sigma(I)^{m} v_{I} \otimes v_{I^{c}} .
$$

Hence if $m$ is even, then $\Delta\left(v_{\mathbb{N}_{N}}\right)=\sum_{I \subset \mathbb{N}_{N}} v_{I} \otimes v_{I^{c}}$, and if $m$ is odd, then $\Delta\left(v_{\mathbb{N}_{N}}\right)=$ $\sum_{I \subset \mathbb{N}_{N}} \sigma(I) v_{I} \otimes v_{I^{c}}$.

Proof We prove the lemma by induction on $N$. For $N=1$ the lemma is an immediate consequence of the fact that $v_{1}$ is primitive. For the induction step we assume that the formula holds for $N-1$. We compute

$$
\begin{aligned}
\Delta\left(v_{\mathbb{N}_{N}}\right)= & \Delta\left(v_{\mathbb{N}_{N-1}} v_{N}\right) \\
= & \Delta\left(v_{\mathbb{N}_{N-1}}\right) \Delta\left(v_{N}\right) \\
= & \left(\sum_{I \subset \mathbb{N}_{N-1}} \sigma(I)^{m} v_{I} \otimes v_{\mathbb{N}_{N-1} \backslash I}\right)\left(1 \otimes v_{N}+v_{N} \otimes 1\right) \\
= & \sum_{I \subset \mathbb{N}_{N-1}} \sigma(I)^{m} v_{I} \otimes\left(v_{\mathbb{N}_{N-1} \backslash I} v_{N}\right) \\
& +\sum_{I \subset \mathbb{N}_{N-1}}(-1)^{\operatorname{deg}\left(v_{\mathbb{N}_{N-1} \backslash I}\right) \operatorname{deg}\left(v_{N}\right)} \sigma(I)^{m}\left(v_{I} v_{N}\right) \otimes v_{\mathbb{N}_{N-1} \backslash I} .
\end{aligned}
$$

Since $\operatorname{deg}\left(v_{N}\right)=m$ and $\operatorname{deg}\left(v_{\mathbb{N}_{N-1} \backslash I}\right)=m(N-1-\# I)$, we have for each $I \subset \mathbb{N}_{N-1}$ that

$$
\begin{aligned}
(-1)^{\operatorname{deg}\left(v_{\mathbb{N}_{N-1} \backslash I}\right) \operatorname{deg}\left(v_{N}\right)} \sigma(I)^{m} & =(-1)^{m^{2}(N-1-\# I)} \sigma(I)^{m} \\
& =(-1)^{m(N-1-\# I)} \sigma(I)^{m} \\
& =\sigma(I \cup\{N\})^{m} .
\end{aligned}
$$

The previous sum therefore becomes

$$
\begin{aligned}
= & \sum_{I \subset \mathbb{N}_{N-1}} \sigma(I)^{m} v_{I} \otimes v_{\mathbb{N}_{N} \backslash I} \\
& +\sum_{I \subset \mathbb{N}_{N-1}} \sigma(I \cup\{N\})^{m} v_{I \cup\{N\}} \otimes v_{\mathbb{N}_{N} \backslash(I \cup\{N\})} \\
= & \sum_{I \subset \mathbb{N}_{N}} \sigma(I)^{m} v_{I} \otimes v_{\mathbb{N}_{N} \backslash I} .
\end{aligned}
$$

This proves the induction step and hence the lemma. 
Proof of Proposition 4.1 Recall that by assumption the vectors $v_{1}, \ldots, v_{N}$ are linearly independent and have all the same degree $m$. Since $\operatorname{deg} v_{I}=m^{\# I}$ it suffices to show that for every $k \leq N$ the vectors $v_{I}$ with $I \subset \mathbb{N}_{N}$ and $\# I=k$ are linearly independent. For $k=1$ this is an assumption. For the induction step we assume this assertion for all $k \leq n-1$ where $n \leq N$. Since the coproduct is linear, it suffices to show that the vectors $\Delta\left(v_{I}\right)$ with $I \subset \mathbb{N}_{N}$, \#I=n are linearly independent. It follows from the induction hypothesis that for every $0<k<n$ the vectors $v_{I} \otimes v_{J}$ with $I, J \subset \mathbb{N}_{N}$ and $\# I=k, \# J=n-k$ are linearly independent. This and the formulas

$$
\Delta\left(v_{I}\right)=\sum_{J \subset I} \sigma(J)^{m} v_{J} \otimes v_{I \backslash J},
$$

that follow from (the proof of) Lemma 4.1, complete the induction step.

\section{Proof of Theorem 1.1}

We shall deduce Theorem 1.1 from Corollary 3.1. We start with saying briefly "who is who" in Corollary 3.1: Let $M$ be a closed connected manifold, and let $\widetilde{M}$ be its universal covering space. We take as $G$ the fundamental group $\pi_{1}(M)$ of $M$, and as $V$ we take the homology $H_{*}(\Omega \widetilde{M} ; \mathbb{F})$ over a suitable field $\mathbb{F}$. The product $\mu$ will be the Pontryagin product given by concatenation of loops, and the coproduct will simply come from the diagonal map $\Omega \widetilde{M} \rightarrow \Omega \widetilde{M} \times \Omega \widetilde{M}, x \mapsto(x, x)$. Now fix a Riemannian metric on $M$. The filtration on $V^{r}$ will be given by taking $|v|$ as the smallest $r$ for which $v$ can be represented by a cycle of based loops of length at most $r$, and for $g \in \pi_{1}(M)$ we take $L(g)$ to be (half of) the length of the shortest curve representing $g$.

We shall show in Sects. 5.1 and 5.2 that with these choices, $(V, \mu, \Delta)$ is a filtered Hopf algebra over $\mathbb{F} G$. In Sect. 5.3 we show that for $M$ of non-finite type with $G=$ $\pi_{1}(M)$ virtually polycyclic, the assumption in Theorem 1.1 that $H_{\mathfrak{m}}(\widetilde{M} ; \mathbb{F})$ is infinitedimensional implies the dimension assumptions on $\oplus V_{i}$ in Corollary 3.1.

\subsection{The Hopf-algebra structure on $H_{*}(\Omega \widetilde{M} ; \mathbb{F})$}

Let $M$ be a closed connected manifold, and let pr: $\widetilde{M} \rightarrow M$ be its universal covering space. Fix $p \in M$ and $\tilde{p} \in \widetilde{M}$ over $p$. The spaces $\Omega_{0} M$ and $\Omega \widetilde{M}$ of contractible continuous loops based at $p$ and $\tilde{p}$, respectively, are canonically identified. Conjugation of loops in $\Omega_{0} M$ by loops in $M$ based at $p$ yields an action of the fundamental group $G=\pi_{1}(M, p)$ on $H_{*}\left(\Omega_{0} M\right)=H_{*}(\Omega \widetilde{M})$ : Given a cycle $C=\{\gamma\}$ of loops in $\Omega \widetilde{M}$ based at $\tilde{p}$ and given $g \in G$, the class $g[C]$ is defined as the class represented by the cycle of loops $\left\{c_{g}^{-1} \circ g \gamma \circ c_{g}\right\}$, where $c_{g}$ is the lift to $\widetilde{M}$ starting at $\tilde{p}$ of a loop in $M$ in class $g$, and $g \gamma$ is the lift of $\operatorname{pr} \circ \gamma$ starting at $g \tilde{p}$.

Let $\mathbb{F}$ be the field, and abbreviate $V_{*}:=H_{*}(\Omega \widetilde{M} ; \mathbb{F})$. The action of $G$ on $V$ extends to an action of $\mathbb{F} G$ on $V$. Concatenation of loops in $\widetilde{M}$ based at $\tilde{p}$ induces a product $\mu: V \otimes V \rightarrow V$, called the Pontryagin product. Since concatenation of loops is associative up to homotopy, $\mu$ is associative. It follows from the definition of the action of $G$ that $\mu$ is $\mathbb{F} G$-linear. In order to define the coproduct $\Delta: V \rightarrow V \otimes V$, we 
consider, more generally, a topological space $X$ and the diagonal map $\delta_{X}: X \rightarrow X \times X$, $x \mapsto(x, x)$. Since we work over a field $\mathbb{F}$, the cross product

$$
H_{*}(X ; \mathbb{F}) \otimes H_{*}(X ; \mathbb{F}) \stackrel{\times}{\longrightarrow} H_{*}(X \times X ; \mathbb{F})
$$

is an isomorphism by the Künneth formula. We can therefore define $\Delta_{X}: H_{*}(X ; \mathbb{F}) \rightarrow$ $H_{*}(X ; \mathbb{F}) \otimes H_{*}(X ; \mathbb{F})$ by

$$
\Delta_{X}:=\times^{-1} \circ\left(\delta_{X}\right)_{*}
$$

Assume now in addition that $X$ is a path-connected $\mathrm{H}$-space, with product $v$.

Lemma 5.1 The homology $H_{*}(X ; \mathbb{F})$, with product induced by $v$ and with coproduct $\Delta_{X}$, is a Hopf algebra.

We refer to [33, Theorem 7.15] for the proof. For the readers convenience, we verify that for every homogeneous element $v$ of positive degree, $\Delta_{X} v$ has the form (3.1). Let $p: X \times X \rightarrow X,(x, y) \mapsto x$, be the projection on the first factor. Then

$$
p \circ \delta_{X}=i d_{X}
$$

For an element $u=v_{n}^{\prime} \otimes 1+1 \otimes v_{n}^{\prime \prime}+\sum v_{i}^{\prime} \otimes v_{j}^{\prime \prime} \in \oplus_{i+j=n} H_{i}(X) \otimes H_{j}(X)$ with $\operatorname{deg} v_{i}^{\prime}<n$ we have

$$
\left(p_{n} \circ \times\right) u=v_{n}^{\prime}
$$

by the geometric definition of the cross product (see e.g. $[15, \S 3 . \mathrm{B}]$ ). Now write $\Delta_{X} v=v_{n}^{\prime} \otimes 1+1 \otimes v_{n}^{\prime \prime}+\sum v_{i}^{\prime} \otimes v_{j}^{\prime \prime}$ with $\operatorname{deg} v_{i}^{\prime}, \operatorname{deg} v_{j}^{\prime \prime} \geq 1$. Using (5.1), the definition of $\Delta_{X}$ and (5.2) we get

$$
\begin{aligned}
v & =p_{n} \circ\left(\delta_{X}\right)_{n} v \\
& =p_{n} \circ \times \circ \Delta_{X} v \\
& =p_{n} \circ \times\left(v_{n}^{\prime} \otimes 1+1 \otimes v_{n}^{\prime \prime}+\sum v_{i}^{\prime} \otimes v_{j}^{\prime \prime}\right) \\
& =v_{n}^{\prime} .
\end{aligned}
$$

Similarly we find $v_{n}^{\prime \prime}=v$, and so $\Delta_{X} v=v \otimes 1+1 \otimes v+\sum v_{i}^{\prime} \otimes v_{j}^{\prime \prime}$ with $\operatorname{deg} v_{i}^{\prime}$, $\operatorname{deg} v_{i}^{\prime \prime} \geq 1$.

Since $\tilde{M}$ is simply connected, $\Omega \widetilde{M}$ is path-connected. Hence $V_{0}=H_{0}(\Omega \widetilde{M} ; \mathbb{F}) \cong$ $\mathbb{F}$ is one-dimensional. Moreover, $1 \in \mathbb{F}$ corresponds to the class of the constant path $\tilde{p} \in \widetilde{M}$, which is the unit for the Pontryagin product $\mu$. Applying Lemma 5.1 with $X=\Omega \widetilde{M}$ and writing $\Delta$ for $\Delta_{\Omega \widetilde{M}}$ we obtain that $(V, \mu, \Delta)$ is a Hopf algebra over $\mathbb{F} G$. (We have already noticed that $\mu$ is $\mathbb{F} G$-linear. The definition of the $G$-actions on $V$ and on $V \otimes V$ shows that also $\Delta$ is $\mathbb{F} G$-linear.) 


\subsection{The filtration on $H_{*}(\Omega \widetilde{M} ; \mathbb{F})$}

Fix a Riemannian metric $\rho$ on $M$, and let $\tilde{\rho}=\operatorname{pr}^{*} \rho$ be the corresponding Riemannian metric on $\widetilde{M}$. Given a piecewise smooth curve $\gamma$ in $\widetilde{M}$, we denote by $\ell(\gamma)$ the length of $\gamma$ with respect to the Riemannian metric $\widetilde{\rho}$. For $r>0$ let $V^{r}$ be the set of homology classes in $V=H_{*}(\Omega \widetilde{M} ; \mathbb{F})$ that can be represented by cycles formed by piecewise smooth loops $\gamma$ based at $\tilde{p}$ with $\ell(\gamma) \leq r$,

$$
V^{r}:=\iota_{*}^{r} H_{*}\left(\Omega^{r} \widetilde{M} ; \mathbb{F}\right)
$$

Then each $V^{r}$ is finite-dimensional (see [20, §16]), $V^{r} \subset V^{s}$ for $r \leq s$ and $V=$ $\bigcup_{r>0} V^{r}$. As in Sect. 3.2 define the value of $v \in V$ by $|v|:=\min \left\{r \mid v \in V^{r}\right\}$. In view of the definition of the Pontryagin product and by the triangle inequality, $|v w| \leq|v|+|w|$ for all $v, w \in V$.

Next, for $g \in G=\pi_{1}(M, p)$ let $\ell(g)$ be the minimal length of a piecewise smooth loop based at $p$ that represents $g$. In other words, $\ell(g)$ is the length of the shortest geodesic lasso based at $p$ in class $g$. Set $L(g):=\frac{1}{2} \ell(g)$. Then $L(g)=L\left(g^{-1}\right)$ and $L(g h) \leq L(g)+L(h)$ for all $g, h \in G$ by the triangle inequality. Finally, for $g \in G$ denote by $\underline{c}_{g}$ the lift to $\widetilde{M}$ based at $\tilde{p}$ of a shortest curve in class $g$. Then

$$
\ell\left(\underline{c}_{g}^{-1} \circ g \gamma \circ \underline{c}_{g}\right) \leq \ell\left(\underline{c}_{g}^{-1}\right)+\ell(g \gamma)+\ell\left(\underline{c}_{g}\right)=\ell(\gamma)+2 \ell\left(\underline{c}_{g}\right)=\ell(\gamma)+L(g)
$$

for all $g \in G$ and $\gamma \in \Omega \widetilde{M}$. In view of the definition of the $G$-action on $\Omega \widetilde{M}$ we find that $|g v| \leq|v|+L(g)=|v|+|g|$. We have shown that $(V, \mu, \Delta)$ is a filtered Hopf algebra over $\mathbb{F} G$.

\subsection{Dimensions}

Recall that $M$ is of finite type if its universal cover $\widetilde{M}$ is homotopy equivalent to a finite CW-complex.

Lemma 5.2 [8, Lemma 2.2] The following are equivalent.

(i) $M$ is of finite type.

(ii) The Abelian groups $H_{k}(\tilde{M})$ are finitely generated for all $k \geq 1$.

(iii) The Abelian groups $\pi_{k}(M)$ are finitely generated for all $k \geq 2$.

Now assume that $M$ is not of finite type. By the lemma, we can define

$$
\mathfrak{m}(M):=\min \left\{k \mid H_{k}(\widetilde{M}) \text { is not finitely generated }\right\} \in\{2, \ldots, \operatorname{dim} M\} .
$$

The main result of this subsection is

Proposition 5.1 Assume that $M$ is not of finite type. Let $\mathfrak{m}=\mathfrak{m}(M)$ be as in definition (5.3). Then

(i) $H_{i}(\Omega \widetilde{M})$ is finitely generated for $i \leq \mathfrak{m}-2$; 
(ii) $H_{\mathfrak{m}-1}(\Omega \widetilde{M})$ is not finitely generated, but is finitely generated as a $\mathbb{Z} G$-module.

Proof For each $k \geq 2$ the fundamental group $G=\pi_{1}(M, p)$ acts on the homotopy group $\pi_{k}(M)=\pi_{k}(M, p)$ by conjugation. Under the Hurewicz homomorphism $h: \pi_{k}(M)=\pi_{k}(\widetilde{M}) \rightarrow H_{k}(\tilde{M})$ this action corresponds to the action of $G$ on $H_{k}(\tilde{M})$ induced by deck transformations (the commutative diagram on the left). On $\pi_{k-1}(\Omega \widetilde{M})=\pi_{k-1}\left(\Omega_{0} M\right) \cong \pi_{k}(M)$ this action of $G$ is induced by conjugation of elements in $\Omega_{0} M \cong \Omega \widetilde{M}$. This action also induces an action on $H_{k-1}(\Omega \widetilde{M})$, namely the action described in Sect. 5.1, and the two actions commute with the Hurewicz homomorphism $h: \pi_{k-1}(\Omega \widetilde{M}) \rightarrow H_{k-1}(\Omega \widetilde{M})$ (the commutative diagram on the right),

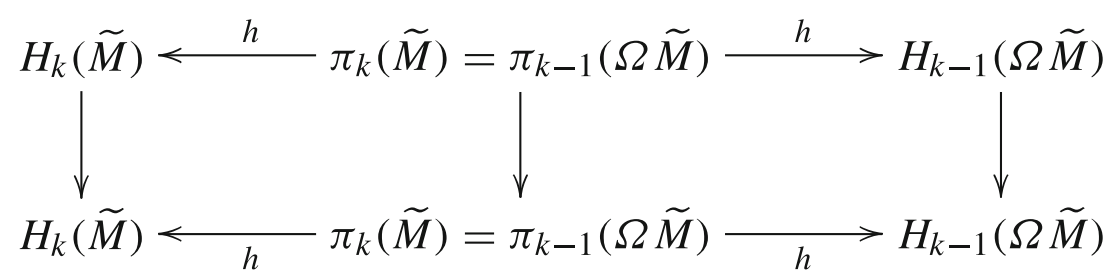

Recall that $\mathfrak{m} \in\{2, \ldots, \operatorname{dim} M\}$ is the minimal integer such that $H_{\mathfrak{m}}(\widetilde{M})$ is not finitely generated. By Serre's theory of $\mathcal{C}$-classes, applied to the class of finitely generated Abelian groups, $\mathfrak{m}$ is also the minimal integer such that $\pi_{\mathfrak{m}}(\widetilde{M})$ is not finitely generated. More precisely, Serre's Hurewicz theorem implies that for $k \leq \mathfrak{m}$ the Hurewicz map $h: \pi_{k}(\widetilde{M}) \rightarrow H_{k}(\widetilde{M})$ is injective and surjective up to finitely generated groups, see [30] or [16, Theorem 1.8]. Hence $\pi_{k}(\Omega \widetilde{M})$ is finitely generated for $k \leq \mathfrak{m}-2$, but not so for $k=\mathfrak{m}-1$.

Since $\Omega \widetilde{M}$ is a path-connected H-space, $\pi_{1}(\Omega \widetilde{M})$ acts trivially on $\pi_{k}(\Omega \widetilde{M})$ for $k \geq 0$. Serre's Hurewicz theorem now implies that the map $h: \pi_{k}(\Omega \widetilde{M}) \rightarrow H_{k}(\Omega \widetilde{M})$ has finitely generated kernel and cokernel for $k \leq \mathfrak{m}-1$, see [30, p. 274] or [16, Theorem 1.8]. It follows that $H_{k}(\Omega \widetilde{M})$ is finitely generated for $k \leq \mathfrak{m}-2$, but not so for $k=\mathfrak{m}-1$.

We are left with proving the second assertion in (ii). After replacing $M$ by a homotopy equivalent space, if necessary, we find a CW-structure on $M$. Since $M$ is compact, this CW-structure is finite. We lift this structure to $\widetilde{M}$ by the action of $G$. The cellular chain complex $C_{*}(\widetilde{M} ; \mathbb{Z})$ is then a finitely generated $\mathbb{Z} G$-module in each degree. Since $G$ is virtually polycyclic, the ring $\mathbb{Z} G$ is left Noetherian, see [14] or [17]. Hence each $\mathbb{Z} G$-module $C_{*}(\widetilde{M} ; \mathbb{Z})$ is left Noetherian. Therefore the kernel and the image of the differential of $C_{*}(\widetilde{M} ; \mathbb{Z})$ as well as the quotient $H_{*}(\widetilde{M})$ are finitely generated left Noetherian $\mathbb{Z} G$-modules in each degree. In particular, $H_{\mathfrak{m}}(\widetilde{M})$ is a finitely generated $\mathbb{Z} G$-module. Recall that $h: \pi_{\mathfrak{m}}(\tilde{M}) \rightarrow H_{\mathfrak{m}}(\widetilde{M})$ is injective and surjective up to finitely generated groups. In view of the commutative diagram above, it follows that $\pi_{\mathfrak{m}}(\widetilde{M})$ and hence $\pi_{\mathfrak{m}-1}(\Omega \widetilde{M})$ are finitely generated $\mathbb{Z} G$-modules. As we have seen before, $h: \pi_{\mathfrak{m}-1}(\Omega \widetilde{M}) \rightarrow H_{\mathfrak{m}-1}(\Omega \widetilde{M})$ has finitely generated cokernel. Hence $H_{\mathfrak{m}-1}(\Omega \widetilde{M})$ is also a finitely generated $\mathbb{Z} G$-module. 


\subsection{Proof of Theorem 1.1 and Remark 1.2 (i)}

By the universal coefficient theorem,

$$
H_{i}(\Omega \widetilde{M} ; \mathbb{F})=H_{i}(\Omega \widetilde{M}) \otimes \mathbb{F} \oplus \operatorname{Tor}\left(H_{i-1}(\Omega \widetilde{M}) ; \mathbb{F}\right)
$$

for every field $\mathbb{F}$. Hence assertion (i) of Proposition 5.1 implies that $H_{i}(\Omega \widetilde{M} ; \mathbb{F})$ is finite-dimensional for $i \leq \mathfrak{m}-2$, and assertion (ii) implies that $H_{\mathfrak{m}-1}(\Omega \widetilde{M} ; \mathbb{F})$ is finitely generated as an $\mathbb{F} G$-module.

Assume now that $\mathbb{F}$ is a field such that $H_{\mathfrak{m}}(\widetilde{M} ; \mathbb{F})$ is infinite-dimensional. Since

$$
H_{\mathfrak{m}}(\tilde{M} ; \mathbb{F})=H_{\mathfrak{m}}(\widetilde{M}) \otimes \mathbb{F} \oplus \operatorname{Tor}\left(H_{\mathfrak{m}-1}(\tilde{M}) ; \mathbb{F}\right)
$$

and since $H_{\mathfrak{m}-1}(\widetilde{M})$ is finitely generated, $H_{\mathfrak{m}}(\widetilde{M}) \otimes \mathbb{F}$ is also infinite-dimensional. As we have seen in the proof above, the two Hurewicz maps

$$
H_{\mathfrak{m}}(\tilde{M}) \longleftarrow h \pi_{\mathfrak{m}}(\tilde{M})=\pi_{\mathfrak{m}-1}(\Omega \widetilde{M}) \stackrel{h}{\longrightarrow} H_{\mathfrak{m}-1}(\Omega \widetilde{M})
$$

both have finitely generated kernel and cokernel. Hence $H_{\mathfrak{m}-1}(\Omega \widetilde{M}) \otimes \mathbb{F}$ is also infinite-dimensional. This and (5.4) imply that $H_{\mathfrak{m}-1}(\Omega \widetilde{M} ; \mathbb{F})$ is infinite-dimensional.

The dimension assumptions in Corollary 3.1 are thus satisfied, and we conclude that $\operatorname{dim} V^{T}$ grows at least like $e^{\sqrt{T}}$.

Similarly, the identity (5.5) shows that $\operatorname{dim} H_{\mathfrak{m}}(\widetilde{M} ; \mathbb{F})=\infty$ if and only if $\operatorname{dim} H_{\mathfrak{m}}(\widetilde{M}) \otimes \mathbb{F}=\infty$, which holds if and only if $\operatorname{dim} \pi_{\mathfrak{m}}(\widetilde{M}) \otimes \mathbb{F}=\infty$ because the left map in (5.6) has finitely generated kernel and cokernel.

\section{A generalization to simplicial complexes, and a "counterexample"}

\subsection{An extension to simplicial complexes}

Our main result Theorem 1.1 holds true for $\mathrm{CW}$-complexes. To avoid some technicalities with rectifiable loops, we restrict ourselves to simplicial complexes, linearly embedded in some Euclidean space.

Consider a finite simplicial complex $K$. Fix a piecewise linear embedding of $K$ into some $\mathbb{R}^{N}$. Then every point $x \in K$ has a neighbourhood $U \subset K$ such that for every $u \in U$ the straight line from $x$ to $u$ lies in $K$ and is strictly shorter than any other rectifiable curve in $K$ from $x$ to $u$. Milnor's method of approximating the loop space by broken geodesics, [20, $\S 16$, now shows that every cycle in $\Omega K$ is homotopic to a cycle in the space of piecewise linear loops in $K$. Hence every homology class in the loop space $\Omega K$ can be represented by a cycle formed by piecewise linear loops.

Denote by $\Omega_{0}^{T} K$ the space of contractible based loops in $K$ that are piecewise linear and of length $\ell(\gamma) \leq T$. For further use we state two other consequences of the 
approximation method in [20, §16] (see also [13, p. 353]): First, each sublevel $\Omega_{0}^{T} K$ is homotopy equivalent to a finite polyhedron, and hence

$$
\iota_{*}^{T} H_{*}\left(\Omega_{0}^{T} K\right) \quad \text { is finitely generated for each } T .
$$

In particular, all vector spaces $\iota_{*}^{T} H_{*}\left(\Omega_{0}^{T} K ; \mathbb{F}\right)$ are finite-dimensional. Second, there exists a constant $c=c(X, \rho)$ such that no $k$-cycle in $\Omega_{0} K$ can be represented by a cycle in $\Omega_{0}^{c k} K$. In other words, for every $k \geq 1$,

$$
\iota_{k}^{T} H_{k}\left(\Omega_{0}^{T} K\right)=0 \quad \text { for } T \leq c k
$$

The proof of Theorem 1.1 applies literally to the present situation:

Theorem 6.1 Let $K$ be a finite connected simplicial complex, embedded into some $\mathbb{R}^{N}$. Assume that $K$ is not of finite type and has virtually polycyclic fundamental group. Assume further that there exists a field $\mathbb{F}$ such that $H_{\mathfrak{m}}(\widetilde{K} ; \mathbb{F})$ is infinitedimensional. Then the function of $T$

$$
\operatorname{dim} \iota_{*}^{T} H_{*}\left(\Omega_{0}^{T} K ; \mathbb{F}\right)
$$

grows at least like $e^{\sqrt{T}}$.

\subsection{A "counterexample"}

We now illustrate the role of the assumption that there exists a field $\mathbb{F}$ such that $H_{\mathfrak{m}}(\widetilde{K} ; \mathbb{F})$ is infinite-dimensional by an example. Let $X$ be the mapping torus of a degree- $p$ map $f$ of the sphere $S^{n}$, where $p$ is prime and $n \geq 2$. Since self-maps of spheres of equal degree are homotopic, $p$ and $n$ determine $X$ up to homotopy equivalence. If we endow $S^{n}$ with the CW-structure with two cells, the 0 -cell $\star$ being a fixed point of $f$, then the attaching map $f$ is cellular, and we obtain a CW-structure on $X$. Since $H_{k}(X)=\mathbb{Z}$ for $k=0,1$ and $H_{k}(X)=0$ otherwise, $X$ is not homotopyequivalent to a topological manifold.

We now compute $H_{*}(\widetilde{X})$ and $H_{*}(\Omega \widetilde{X})$. The fundamental group of $X$ is infinite cyclic, $\pi_{1}(X)=\mathbb{Z}$. The universal cover $\widetilde{X}$ is the "double mapping telescope" obtained by glueing together the mapping cylinders of $f_{i}=f, i \in \mathbb{Z}$. For integers $i \leq j$ consider the part of $\widetilde{X}$ over $[i, j]$,

$$
\widetilde{X}_{[i, j]}=\{(x, t) \in \widetilde{X} \mid t \in[i, j]\}
$$

and write $\widetilde{X}_{j}=\widetilde{X}_{[j, j]}$. By moving along $t$ we see that $\widetilde{X}_{[i, j]}$ deformation retracts onto $\widetilde{X}_{j}=S^{n}$. Retract $\widetilde{X}_{[-1,0]}$ to $\widetilde{X}_{0}$ in time $\left[0, \frac{1}{2}\right]$. Then $\widetilde{X}_{[-2,-1]} \subset \widetilde{X}$ becomes $\widetilde{X}_{[-1,0]}$; retract it to $\widetilde{X}_{0}$ in time $\left[\frac{1}{2}, \frac{3}{4}\right]$. Going on this way we construct a deformation retraction $\widetilde{X} \rightarrow \widetilde{X}_{\geq 0}$ onto the mapping telescope formed by the mapping cylinders of $f_{i}=f, i \geq 0$. 
Since $f$ has degree $p$, the inclusion $\widetilde{X}_{[0, j]} \rightarrow \widetilde{X}_{[0, j+1]}$ induces multiplication by $p$ in homology,

$$
H_{n}\left(\tilde{X}_{[0, j]}\right)=\mathbb{Z} \stackrel{p}{\longrightarrow} \mathbb{Z}=H_{n}\left(\widetilde{X}_{[0, j+1]}\right)
$$

The direct limit of the sequence $\mathbb{Z} \stackrel{p}{\longrightarrow} \mathbb{Z} \stackrel{p}{\longrightarrow} \mathbb{Z} \longrightarrow \cdots$ can be identified with $\mathbb{Z}[1 / p]$, the subgroup of $\mathbb{Q}$ consisting of rational numbers with denominators a power of $p$. Hence

$$
H_{n}(\tilde{X})=H_{n}\left(\widetilde{X}_{\geq 0}\right)=\lim _{\longrightarrow} H_{n}\left(\widetilde{X}_{[0, j]}\right) \cong \mathbb{Z}[1 / p],
$$

(cf. Example 3.F.3 in [15]), and $H_{k}(\widetilde{X})=0$ for all other $k \geq 1$. The Abelian group $\mathbb{Z}[1 / p]$ is not finitely generated, hence $\mathfrak{m}=n$ in this example. However, for a field $\mathbb{F}$ of characteristic $q$ we have $H_{n}(\widetilde{X} ; \mathbb{F})=0$ if $q=p$ and $H_{n}(\widetilde{X} ; \mathbb{F}) \cong \mathbb{F}$ otherwise.

We next compute $H_{*}(\Omega \widetilde{X})$. We choose as base point of $\widetilde{X}$ the point $\star \in \widetilde{X}_{0} \cong S^{n}$. Let $\mathfrak{p}$ be the path from $\star \in \widetilde{X}_{0}$ to $(\star, j) \in \widetilde{X}_{j}$ traced by $\star$ under the canonical deformation retraction $\widetilde{X}_{[0, j]} \rightarrow \widetilde{X}_{j}$. The space $\left(\Omega \widetilde{X}_{[0, j]}, \star\right)$ is homeomorphic to the subspace of $\left(\Omega \widetilde{X}_{[0, j]},(\star, j)\right)$ obtained by conjugating by $\mathfrak{p}$ the loops in $\left(\Omega \widetilde{X}_{[0, j]},(\star, j)\right)$. Under the deformation retraction $\widetilde{X}_{[0, j]} \rightarrow \widetilde{X}_{j}$ this subspace deformation retracts onto $\left(\Omega \widetilde{X}_{j},(\star, j)\right)=\left(\Omega S^{n}, \star\right)$. We have

$$
H_{k}\left(\Omega S^{n}\right)= \begin{cases}\mathbb{Z} & \text { if } k \in(n-1) \mathbb{N} \cup 0 \\ 0 & \text { otherwise. }\end{cases}
$$

Fix $k=(n-1) \ell$. The natural homotopy equivalence $J\left(S^{n-1}\right) \rightarrow \Omega S^{n}$ from the James reduced product of $S^{n-1}$ to $\Omega S^{n}$ shows that the map $\Omega f: \Omega S^{n} \rightarrow \Omega S^{n}$ induces multiplication by $p^{\ell}$ in $H_{k}$,

$$
H_{k}\left(\Omega S^{n}\right)=\mathbb{Z} \stackrel{p^{\ell}}{\longrightarrow} \mathbb{Z}=H_{k}\left(\Omega S^{n}\right)
$$

see $[18]$ or $[15, \S 4 . J]$. The direct limit of the sequence $\mathbb{Z} \stackrel{p^{\ell}}{\longrightarrow} \mathbb{Z} \stackrel{p^{\ell}}{\longrightarrow} \mathbb{Z} \longrightarrow \cdots$ can be identified with $\mathbb{Z}\left[1 / p^{\ell}\right]$, the subgroup of $\mathbb{Q}$ consisting of rational numbers with denominators a power of $p^{\ell}$. Hence

$$
H_{k}(\Omega \widetilde{X})=H_{k}\left(\Omega \widetilde{X}_{\geq 0}\right)=\lim _{\rightarrow} H_{k}\left(\Omega \widetilde{X}_{[0, j]}\right) \cong \mathbb{Z}\left[1 / p^{\ell}\right] .
$$

Moreover, $H_{k}(\Omega \widetilde{X})=0$ if $k \notin(n-1) \mathbb{N} \cup 0$.

Every finite $\mathrm{CW}$-complex is homotopy equivalent to a finite simplicial complex, see [32, Theorem 24]. So choose a finite simplicial complex $K$ homotopy equivalent to $X$, and fix an embedding of $K$ into some Euclidean space.

Proposition 6.1 The space $K$ fulfills all assumptions of Theorem 6.1 with $\mathfrak{m}=n$, besides for $H_{\mathfrak{m}}(\widetilde{K} ; \mathbb{F})$ being zero- or one-dimensional for every field $\mathbb{F}$. Moreover, $\iota_{*}^{T} H_{*}\left(\Omega_{0}^{T} K\right)$ is a free Abelian group whose rank grows not more than linearly with $T$. 
Proof Since $X$ and $K$ are homotopy equivalent, so are $\widetilde{X}$ and $\widetilde{K}$. The homologies of $\widetilde{X}$ and $\widetilde{K}$ are thus isomorphic, and so are the homologies of $\Omega \widetilde{X}$ and $\Omega \widetilde{K}$. It remains to understand the growth of $\iota_{*}^{T} H_{*}\left(\Omega^{T} \widetilde{K}\right)$. If $k \notin(n-1) \mathbb{N} \cup 0$, then $\iota_{k}^{T} H_{k}\left(\Omega^{T} \widetilde{K}\right) \subset H_{k}(\Omega \widetilde{K})=0$, so $\iota_{k}^{T} H_{k}\left(\Omega^{T} \widetilde{K}\right)=0$. If $k=(n-1) \ell$, then $\iota_{k}^{T} H_{k}\left(\Omega^{T} \widetilde{K}\right) \subset H_{k}(\Omega \widetilde{K})=\mathbb{Z}\left[1 / p^{\ell}\right]$. By (6.1), the group $\iota_{k}^{T} H_{k}\left(\Omega^{T} \widetilde{K}\right)$ is finitely generated. Every finitely generated subgroup of $\mathbb{Z}\left[1 / p^{\ell}\right]$ is contained in the subgroup generated by some element $1 / p^{i \ell}$ and hence is trivial or infinite cyclic. Moreover, by (6.2) there exists a constant $c$ depending only on $K$ and $\rho$ such that $\iota_{k}^{T} H_{k}\left(\Omega^{T} \widetilde{K}\right)=0$ for $T \leq c k$. Hence $\iota_{*}^{T} H_{*}\left(\Omega^{T} \widetilde{K}\right)$ is a free Abelian group whose rank grows not more than linearly with $T$.

\section{Proof of Corollaries 1.1 and 1.2}

Proof of Corollary 1.1 Let $\mathcal{C}$ be a component of $\Omega_{p q} M$. Fix a smooth path $c \in \mathcal{C}$, of length $\ell(c)$. The map $h: \Omega_{0}(M, p) \rightarrow \mathcal{C}, \gamma \mapsto c \circ \gamma$ is a homotopy equivalence. It maps $\Omega_{0}^{T} M$ to $\mathcal{C}^{T+\ell(c)}$, where $\mathcal{C}^{T}$ is the space of piecewise smooth paths in $\mathcal{C}$ of length $\leq T$. Theorem 1.1 now implies that

$$
\operatorname{dim} \iota_{*}^{T} H_{*}\left(\mathcal{C}^{T} ; \mathbb{F}\right) \text { grows at least like } e^{\sqrt{T}}
$$

Notice that $\operatorname{dim} \iota_{*}^{T} H_{*}\left(\mathcal{C}^{T} ; \mathbb{F}\right) \leq \operatorname{dim} H_{*}\left(\mathcal{C}^{T} ; \mathbb{F}\right)$. For geodesic flows, Corollary 1.1 now follows from classical Morse theory, see [20, Theorem 16.3] or [25, p. 116]. For the general case of Reeb flows, we use (7.1) and Lagrangian Floer homology, exactly as in [19, Section 6]. We outline the argument, omitting all technical issues.

Let $\Sigma \subset T^{*} M$ be the fiberwise starshaped hypersurface corresponding to the cooriented contact manifold ( $S^{*} M, \alpha$ ), and (up to the time change $t \mapsto 2 t$ ) view the Reeb flow $\varphi_{\alpha}^{t}$ on $\left(S^{*} M, \alpha\right)$ as the restriction of the Hamiltonian flow $\varphi_{K}^{t}$ on $T^{*} M$, where $\Sigma=K^{-1}(1)$ and $K$ is fiberwise homogeneous of degree 2.

Denote by $\mathcal{P}(K, \mathcal{C})$ the set of smooth paths $x:[0,1] \rightarrow T^{*} M$ with $x(0) \in \Sigma_{p}$, $x(1) \in \Sigma_{q}$ that are solutions to Hamilton's equation $\dot{x}(t)=X_{K}(x(t))$ and whose projections to $M$ belong to $\mathcal{C}$. The action of $x \in \mathcal{P}(K, \mathcal{C})$ is

$$
\mathcal{A}_{K}(x)=\int_{0}^{1}(\lambda(\dot{x}(t))-K(x(t))) d t
$$

where $\lambda$ is the Liouville 1 -form on $T^{*} M$. Since $K$ is homogenous of degree two, the set $\mathcal{P}^{T^{2} / 2}(K, \mathcal{C})=\left\{x \in \mathcal{P}(K, \mathcal{C}) \mid \mathcal{A}_{K}(x) \leq T^{2} / 2\right\}$ is in bijection with the Reeb chords from $\Sigma_{p}$ to $\Sigma_{q}$ of time $\leq T$. We thus wish to show that $\# \mathcal{P}^{T^{2} / 2}(K, \mathcal{C})$ grows at least like $e^{\sqrt{T}}$.

Set $a=T^{2} / 2$. Since $p, q$ are non-conjugate for the Reeb flow $\varphi_{\alpha}^{t}$, the set $\mathcal{P}^{a}(K, \mathcal{C})$ is finite. The Floer chain group $\mathrm{CF}^{a}(K, \mathcal{C})$ is the $\mathbb{F}$-vector space freely generated by the chords in $\mathcal{P}^{a}(K, \mathcal{C})$. The Conley-Zehnder index of these chords (normalized such that it agrees with the Morse index in case of a non-degenerate geodesic chord) gives 
this vector space a grading $*$. The Floer boundary operator on $\mathrm{CF}_{*}^{a}(K, \mathcal{C})$ is a map of degree -1 . Its homology is the Floer homology $\mathrm{HF}_{*}^{a}(K, \mathcal{C})$. Since the dimension of the homology $\mathrm{HF}_{*}^{a}(K, \mathcal{C})$ is not greater than the dimension of the chain group $\mathrm{CF}_{*}^{a}(K, \mathcal{C})$, which equals $\# \mathcal{P}^{a}(K, \mathcal{C})$, we wish to show that $\operatorname{dim}_{*} \mathrm{HF}_{*}^{a}(K, \mathcal{C})$ grows at least like $e^{\sqrt{T}}$.

Fix a Riemannian metric $g$ on $M$, and let $G: T^{*} M \rightarrow \mathbb{R}$ be the corresponding geodesic Hamiltonian. After multiplying $g$ with a constant, we can assume that $G \leq K$, and since $\Sigma$ is fiberwise starshaped with respect to the origin, we find a constant $\sigma \geq 1$ such that

$$
G \leq K \leq \sigma G
$$

The Floer homology of the functions $G$ and $\sigma G$ is defined in the same way.

There is a commutative diagram

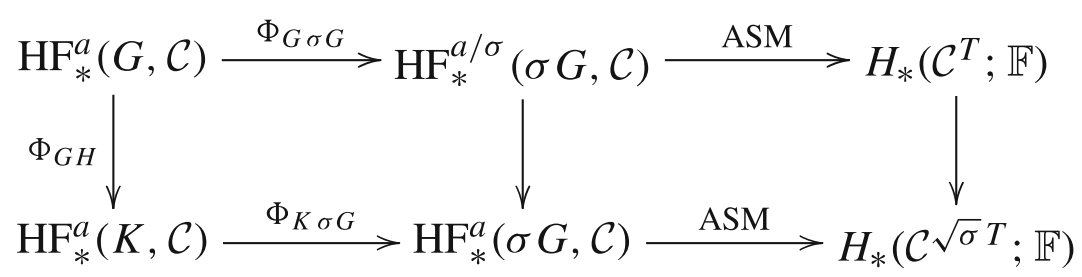

Here, the three maps $\Phi$ between Floer homologies are Floer continuation maps, and $\Phi_{G \sigma G}$ is an isomorphism. The upper map ASM is the composition

$$
\mathrm{HF}_{*}^{a / \sigma}(\sigma G, \mathcal{C}) \stackrel{\mathrm{AS}}{\longrightarrow} \mathrm{HM}_{*}^{a / \sigma}(L, \mathcal{C}) \stackrel{\mathrm{AM}}{\longrightarrow} H_{*}\left(\mathcal{C}^{T} ; \mathbb{F}\right)
$$

of the Abbondandolo-Schwarz isomorphism AS from Floer homology to the Morse homology of the Legendre transform $L$ of $\sigma G$ with the Abbondandolo-Majer isomorphism AM from this homology to the homology of $\mathcal{C}^{T}$, see [1] and [2]. Finally, the two unlabeled vertical arrows are induced by inclusion.

It follows that $\operatorname{dim}_{\mathrm{HF}_{*}^{a}}^{a}(K, \mathcal{C})$ is at least the rank of the right vertical map. This rank is at least $\operatorname{dim} \iota_{*}^{T} H_{*}\left(\mathcal{C}^{T} ; \mathbb{F}\right)$, which by (7.1) grows at least like $e^{\sqrt{T}}$.

Proof of Corollary 1.2 For geodesic flows the claim follows from Corollary 1.1 and the geometric arguments in [25, Section 3.1]. For the general case of Reeb flows we use Theorem 1.1 and the idea from [6], that was further developed in [7,8,19]. The proof goes exactly as the proof of Theorem 4.6 in [19]; we therefore again only sketch the proof.

For $q \in M$ consider $\Sigma_{q}=\Sigma \cap T_{q}^{*} M$ and the bounded component $D_{q}$ of $T_{q}^{*} M \backslash \Sigma_{q}$. The "spheres" $\Sigma_{q}$ are Legendrian and the "discs" $D_{q}$ are Lagrangian. Fix $n \in \mathbb{N}$, and take $q \in M$ not conjugate to $p$ for the flow $\varphi_{\alpha}^{t}$. Recall from the previous proof that the generators of the Floer chain groups $\mathrm{CF}_{*}^{n^{2} / 2}(K)$ (where we now do not restrict to a component $\mathcal{C}$ ) are in bijection with the Reeb chords from $\Sigma_{p}$ to $\Sigma_{q}$ of time $\leq n$. This set is in bijection with the intersection points of the Lagrangian discs $\varphi_{K}^{n}\left(D_{p}\right)$ and $D_{q}$. By the previous proof, this number grows at least like $e^{\sqrt{n}}$, uniformly in $q$. 
Since this holds for almost every $q \in M$, the volume of the discs $\varphi_{K}^{n}\left(D_{p}\right)$ grows at least like $e^{\sqrt{n}}$. We refer to Section 4 of [19] for details. Finally, the volume of $\varphi_{K}^{n}\left(\Sigma_{p}\right)$ also grows like $e^{\sqrt{n}}$ in view of Proposition 4.3 in [8].

Acknowledgments We wish to thank Kenji Fukaya for suggesting to us to bring into play Serre's Hurewicz theorem, and we thank Andrew Ranicki for his explanations on splitting obstructions. We are grateful to the referees for their suggestions and their careful reading. The present work is part of the author's activities within CAST, a Research Network Program of the European Science Foundation.

\section{References}

1. Abbondandolo, A., Majer, P.: Lectures on the Morse complex for infinite-dimensional manifolds. Morse theoretic methods in nonlinear analysis and in symplectic topology, pp. 1-74, NATO Sci. Ser. II Math. Phys. Chem., vol. 217. Springer, Dordrecht (2006)

2. Abbondandolo, A., Schwarz, M.: On the Floer homology of cotangent bundles. Commun. Pure Appl. Math. 59, 254-316 (2006)

3. Andrews, G.: The Theory of Partitions. Cambridge University Press, Cambridge (1976)

4. Bott, R., Samelson, H.: On the Pontryagin product in spaces of paths. Comment. Math. Helv. 27(1953), 320-337 (1954)

5. Dinaburg, E.I.: A connection between various entropy characterizations of dynamical systems. Izv. Akad. Nauk SSSR Ser. Mat. 35, 324-366 (1971)

6. Frauenfelder, U., Schlenk, F.: Volume growth in the component of the Dehn-Seidel twist. Geom. Funct. Anal. 15, 809-838 (2005)

7. Frauenfelder, U., Schlenk, F.: Fiberwise volume growth via Lagrangian intersections. J. Symplectic Geom. 4, 117-148 (2006)

8. Frauenfelder, U., Labrousse, C., Schlenk, F.: Slow volume growth for Reeb flows on spherizations and contact Bott-Samelson theorems. arXiv:1307.7290

9. Grigorchuk, R.: Groups of intermediate growth: an introduction. Enseign. Math. 54, 251-272 (2008)

10. Grigorchuk, R.: On the gap conjecture concerning group growth. Bull. Math. Sci. 4, 113-128 (2014)

11. Gromov, M.: Homotopical effects of dilatation. J. Differ. Geom. 13, 303-310 (1978)

12. Gromov, M.: Groups of polynomial growth and expanding maps. Inst. Hautes Études Sci. Publ. Math. 53, 53-73 (1981)

13. Gromov, M.: Metric structures for Riemannian and non-Riemannian spaces. Modern Birkhäuser Classics. Birkhäuser, Boston (2007)

14. Hall, P.: Finiteness conditions for soluble groups. Proc. Lond. Math. Soc. 4, 419-436 (1954)

15. Hatcher, A.: Algebraic Topology. Cambridge University Press, Cambridge (2002)

16. Hatcher, A.: Spectral Sequences in Algebraic Topology. http://www.math.cornell.edu/ hatcher

17. Ivanov, S.V.: Group rings of Noetherian groups. Mat. Zametki 46(6), 61-66, 127 (1989) [translation in Math. Notes 46(5-6), 929-933 (1990)]

18. James, I.M.: Reduced product spaces. Ann. Math. 62, 170-197 (1955)

19. Macarini, L., Schlenk, F.: Positive topological entropy of Reeb flows on spherizations. Math. Proc. Camb. Philos. Soc. 151, 103-128 (2011)

20. Milnor, J.: Morse Theory. Annals of Mathematics Studies, vol. 51. Princeton University Press, Princeton (1963)

21. Nathanson, M.: Elementary methods in number theory. Graduate Texts in Mathematics, vol. 195 (2000)

22. Newhouse, S.: Entropy and volume. Ergodic Theory Dynam. Systems $8^{*}$ (1988), Charles Conley Memorial Issue, pp. 283-299

23. Ozols, V.: Largest normal neighborhoods. Proc. Am. Math. Soc. 61(1976), 99-101 (1977)

24. Paternain, G.: Topological entropy for geodesic flows on fibre bundles over rationally hyperbolic manifolds. Proc. Am. Math. Soc. 125, 2759-2765 (1997)

25. Paternain, G.: Geodesic flows. Progress in Mathematics, vol. 180. Birkhäuser, Boston (1999)

26. Paternain, G., Petean, J.: Entropy and collapsing of compact complex surfaces. Proc. Lond. Math. Soc. 89, 763-786 (2004)

27. Paternain, G., Petean, J.: Zero entropy and bounded topology. Comment. Math. Helv. 81, 287-304 (2006) 
28. Ranicki, A.: Exact sequences in the algebraic theory of surgery. Mathematical Notes, vol. 26. Princeton University Press/University of Tokyo Press, Princeton/Tokyo (1981)

29. Scott, W.: Group Theory. Prentice-Hall, Englewood Cliffs (1964)

30. Serre, J.-P.: Groupes d'homotopie et classes de groupes abéliens. Ann. Math. 58, 258-294 (1953)

31. Wang, D., Zhang, J., Zhuang, G.: Lower bounds of growth of Hopf algebras. Trans. Am. Math. Soc. 365, 4963-4986 (2013)

32. Whitehead, J.H.C.: A certain exact sequence. Ann. Math. 52, 51-110 (1950)

33. Whitehead, G.W.: Elements of homotopy theory. Graduate Texts in Mathematics, vol. 61. Springer, New York (1978)

34. Wolf, J.: Growth of finitely generated solvable groups and curvature of Riemannian manifolds. J. Differ. Geom. 2, 421-446 (1968)

35. Yomdin, Y.: Volume growth and entropy. Israel J. Math. 57, 285-300 (1987) 